\title{
E6 and $E 7$ gene polymorphisms in human papillomavirus Type-6 identified in Southwest China
}

\author{
Zuyi Chen ${ }^{1,2+}$, Qiongyao $\mathrm{Li}^{2,3+}$, Jian Huang ${ }^{1}$, Jin Li ${ }^{4}$, Feng Yang ${ }^{3}$, Xun Min ${ }^{1 *}$ and Zehui Chen ${ }^{1}$
}

\begin{abstract}
Background: Human papillomavirus type-6 (HPV6) is the major etiological agent of anogenital warts both men and women. The present study aimed to characterize the genetic diversity among HPV6 in Southwest China, and to investigate the origin of, selective pressure experienced by, and impact of the resultantly identified genetic variants on the HPV6 secondary structure.
\end{abstract}

Methods: Phylogenetic trees were constructed by Maximum-likelihood and the Kimura 2-parameters methods by Molecular Evolutionary Genetics Analysis version 6.0. The diversity of secondary structure was analyzed by PSIPred software. The selection pressures acting on the E6/E7 genes were estimated by Phylogenetic Analyses by Maximum Likelihood version 4.8 software.

Results: HPV6 was the most prevalent low risk HPV type in southwest China. In total, 143 E6 and E7 gene sequences of HPV6 isolated from patients were sequenced and compared to GenBank HPV6 reference sequence X00203. The results of these analyses revealed that both the HPV6 E6 and E7 were highly conserved within the analyzed patient samples, and comprised only 3 types of variant sequence, respectively. Furthermore, the analysis of HPV6 E6 and E7 sequences revealed seven/five single-nucleotide mutations, two/four and five/one of which were non-synonymous and synonymous, respectively. The phylogenetic analyses of the $E 6$ and $E 7$ sequences indicated that they belonged to sub-lineage A1 and sub-lineage B1, whereas the selective pressure analyses showed that only the E7 mutation sites 4R, 34E, and 52F were positive selection.

Conclusions: HPV6 (detection rate $=13.10 \%$ ) was very prevalent in southwest China, both the HPV6 E6 and E7 sequences were highly conserved within the analyzed patient samples in southwest China, indicating that the low risk HPV6 can adapt to the environment well without much evolution.

Keywords: HPV6, Genetic diversity, E6, E7, Positive selection

\section{Introduction}

Human papillomaviruses (HPVs) are associated with a variety of epithelial lesions, including benign genital warts and cervical intraepithelial neoplasia [1]. To date, more than $250 \mathrm{HPV}$ types have been identified and each of these genotypes are associated with infection at particular anatomical sites. HPV6 may be the most prevalent low risk alpha-papillomavirus type and is commonly associated with genital warts [2]. For example, anogenital

\footnotetext{
* Correspondence: minxunzmu@163.com

${ }^{\dagger}$ Zuyi Chen and Qiongyao Li contributed equally to this work.

'Department of Laboratory medicine, Affiliated Hospital of Zunyi Medical

University, Zunyi, Guizhou, People's Republic of China

Full list of author information is available at the end of the article
}

warts are primarily caused by HPV6 (family Papillomaviridae, genus Alphapapillomavirus, species 10) [3], which brings a significant burden to both the healthcare system and patients. Similarly, one third of Dutch primary school children have cutaneous warts, of which approximately $20 \%$ seek medical treatment each year [4]. Generally speaking, these infections are classified as "not carcinogenic" or "low risk", they often attract negative attention, and thereby cause significant psychological distress [5]. However, some HPV6 variants are classified as "carcinogenic", because they cause infections that lead to potentially fatal conditions, such as tonsillar, and

(C) The Author(s). 2019 Open Access This article is distributed under the terms of the Creative Commons Attribution 4.0 International License (http://creativecommons.org/licenses/by/4.0/), which permits unrestricted use, distribution, and 
malignant laryngeal carcinoma and/or malignant laryngeal papilloma [6-9].

To date, extensive research has been conducted to investigate sequence variation among carcinogenic HPV types; nevertheless, only limited data is available regarding HPV6 variants, despite their significant impact on human health. Structurally, the HPV is a doublestranded, circular DNA virus that encodes E1, E2, E4, E5, E6, E7, L1 and L2 proteins [10]. HPVs infect cells via the basal layer of the stratified epithelium, and viral gene expression is closely linked to the endogenous differentiation program of the host cells [11]. Of the HPVencoded proteins, E6 and E7 have been shown to be the most important pathogenic HPV proteins. They have been previously shown to function as oncoproteins that critically regulate HPV-induced tumorigenesis [12]. Furthermore, they have also been demonstrated to be essential to maintain the extrachromosomal forms of HPV in undifferentiated basal cells [13].

Genetic variability analyses have proven essential to facilitate an improved understanding of the evolution of the papillomavirus. A number of carcinogenic variants have been identified in HPV variants isolated from populations in Southwest China; however, only limited research has been conducted to identify low risk HPV variants. Thus, the present study aimed to analyze E6 and $E 7$ sequence variability among HPV6 isolated from cervical papilloma samples collected from patients in Southwest China. Phylogenetic analyses were conducted to compare the identified nucleotide sequences with those previously described in other ethnic populations. In addition, the secondary structure of the identified sequences were predicted to assess the probably impact of the low risk variants on overall viral function. The results of the study could provide important data for the research on HPV6 prevention, diagnostic, therapeutic and even the design of therapeutic vaccines based on proteins E6 and E7 in Southwest China.

\section{Methods \& Materials}

\section{Clinical samples and HPV typing}

From May 8, 2013 to June 1, 2016, cervical swabs were obtained from patients (with informed consent and ethical approval) at the Affiliated Hospital of Zunyi Medical University, Angel Women's and Children's Hospital, Sichuan Reproductive Health Research Centre Affiliated Hospital, and the Chengdu Western Hospital Maternity Unit. Women over 18 years old with visible cervical lesions and/or HPV-related diseases (e.g. cervical papilloma) were eligible for inclusion. Specimens were stored at $-20^{\circ} \mathrm{C}$ until DNA extraction and HPV typing. Specimens DNA were extracted and tested using the Human Papillomavirus Genotyping Kit For 23 Types (PCR-RDB, reverse Dot Blot) according to the manufacturer's instructions (Yaneng Bio, Shenzhen, China). This kit enabled the classification of the 23 HPV types (HPV 16, $18,31,33,35,39,45,51,52,53,56,58,59,66,68,73$, 83, MM4, 6, 11, 42, 43, and 44).

\section{PCR amplification}

In total, 216 samples were subjected to PCR amplification with $E 6$ and $E 7$ gene primers (see Additional file 1) that were designed using Primer Premier 5.0 software (Premier Biosoft, California, USA) and the HPV6 reference sequences (X00203) listed in the GenBank database (https://www.ncbi.nlm.nih.gov/genbank/). The reaction mixture comprised $2.5 \mathrm{mM}$ dNTPs, $2 \mathrm{U}$ Taq DNA polymerase, 90 pmol of each primer, and sufficient $10 \times \mathrm{Mg}^{2+}$ PCR buffer to reach a final volume of $25 \mu \mathrm{L}$. Amplification of the sample sequences was achieved using thermal cycling conditions comprising an initial denaturation step $\left(5 \mathrm{~min}\right.$ at $\left.95^{\circ} \mathrm{C}\right)$, followed by 39 amplification cycles (each consisting of a $45 \mathrm{~s}$ denaturation step at $94{ }^{\circ} \mathrm{C}$, a $60 \mathrm{~s}$ annealing step at $53^{\circ} \mathrm{C}$, and a $60 \mathrm{~s}$ elongation step at $72^{\circ} \mathrm{C}$ ). All amplified HPV6 E6 and E7 DNA products were detected via electrophoresis using an agarose gel that was impregnated with ethidium bromide, and sequenced using Sanger sequencing (Sango Biotech, Shanghai, China), and all the data were confirmed by repeating the PCR amplification and sequence analysis at least twice.

\section{Sequence analysis}

The secondary structures of the identified sequences were predicted with PSIPRED online software v.3.2 (http://bioinf.cs.ucl.ac.uk/psipred/), using default parameters. This software uses a very stringent cross-validation technique to achieve an average Q3 score of 81.6\% [14]. Any mutation observed to occur with a frequency of $\geq 10 \%$ was considered to be a major mutation. The sequences and variants were subsequently analyzed with various other software programs, including NCBI BLAST (https://blast.ncbi.nlm.nih.gov/Blast.cgi), Primer Premier 5 (Premier Biosoft), and DNAMAN version 5.2.2 (Lynnon Biosoft, California, USA). HPV6 nucleotide positions were numbered in accordance with the GenBank HPV6 reference sequence (X00203).

\section{Phylogenetic analysis of the identified HPV6 variants}

"Maximum-likelihood" phylogenetic trees were constructed for the identified $E 6$ and $E 7$ variants using MEGA v.6 software (http://www.megasoftware.net/home) to apply Kimura's two-parameter model. Tree topology was evaluated by bootstrap resampling 1000 times [15]. The reference viral sequences used to construct the distinct phylogenetic branches were collected from the GenBank database. Numbers above the branches indicate the bootstrap values that are greater than $60 \%$. 


\section{Selective pressure analysis}

Sites within the E6 and E7 HPV6 gene sequences that were likely to be subject to positive selection were predicted by using PAML 4.8 software (codeml tool; http:// abacus.gene.ucl.ac.uk/software/paml.html) to perform likelihood ratio tests (LRTs) to infer nonsynonymous/synonymous nucleotide divergence, according to the method described by Nei and Gojobor [16-18]. The E6 and E7 protein sequences were aligned using ClustalX v. 2.1 software (ftp://ftp.ebi.ac.uk/pub/software/clustalw2/) [19].

\section{Cross-sectional study}

The sequence results were compared to reference sequences in NCBI (https://www.ncbi.nlm.nih.gov/nuccore/). Seven reference sequences were obtained (KU298876; HE599232; FR751328; FR751337; AF092932; L41216; JN252318).

\section{Results}

\section{Characteristics of HPV6 prevalence in Southwest China}

Nine thousand, three hundred and forty nine specimens (mean age $31.56 \pm 8.42$ years old) were collected. The overall positive rate of detectable $23 \mathrm{HPV}$ types was 33.33\% (3116/9349). 22.01\% (2058/9349) specimens were high risk HPV types and 11.32\% (1058/9349) were low risk HPV types. In all HPV-positive women involved in the study, the most common detected HPV types were HPV16 (detection rate $=13.12 \%$ ) and HPV6 (detection rate $=13.10 \%)($ Fig. 1). HPV6 was the most prevalent low risk HPV, followed by HPV11 (265, 8.50\%), HPV-43 (234, 7.50\%) and HPV42 (152, 4.87\%). No HPV59 and HPV44 types were detected in the patients tested.

\section{E6 sequence variations}

In total, 143 HPV6 E6 gene sequences were obtained from the analyzed patient specimens using Sanger sequencing. A comparison of these with the GenBank HPV6 reference sequence (X00203) identified seven polymorphic sites, two of which comprised non-synonymous (D14Y, H50Q), and five of which comprised synonymous mutations. One of the identified non-synonymous mutations, D14Y, occurred within a region that encodes the alpha helix, whereas three of the synonymous mutations occurred in regions encoding the $\alpha$ helix or $\beta$-strand. Notably, the mutation A120T and G372A were discovered in all specimens (Table 1). The table is designed with the reference to previous study $[20,21]$. No insertion or deletion mutations, nor any evidence of introduced premature stop codons, were detected within the analyzed E6 HPV6 sequences. The conducted alignment of the analyzed (453 bp) E6 sequences with reference sequences belonged to sublineage A1 (70.63\%) and sub-lineage B1 (29.37\%) (Fig. 2).

\section{E7 sequence variations}

Compared with the HPV6 reference sequence (X00203), five single-nucleotide changes were identified within the 297 bp E7 open reading frame (ORF), of which four substitutions were non-synonymous, and one substitutions were synonymous. The most common non-synonymous mutation, T155A, occurred with a frequency of $34.27 \%$, resulted in the amino acid (AA) change of F52Y (Phe to Tyr). The C294A was the most common synonymous mutation, and occurred with a frequency of $34.26 \%$. One non-synonymous mutation (F52Y) occurred in the E7

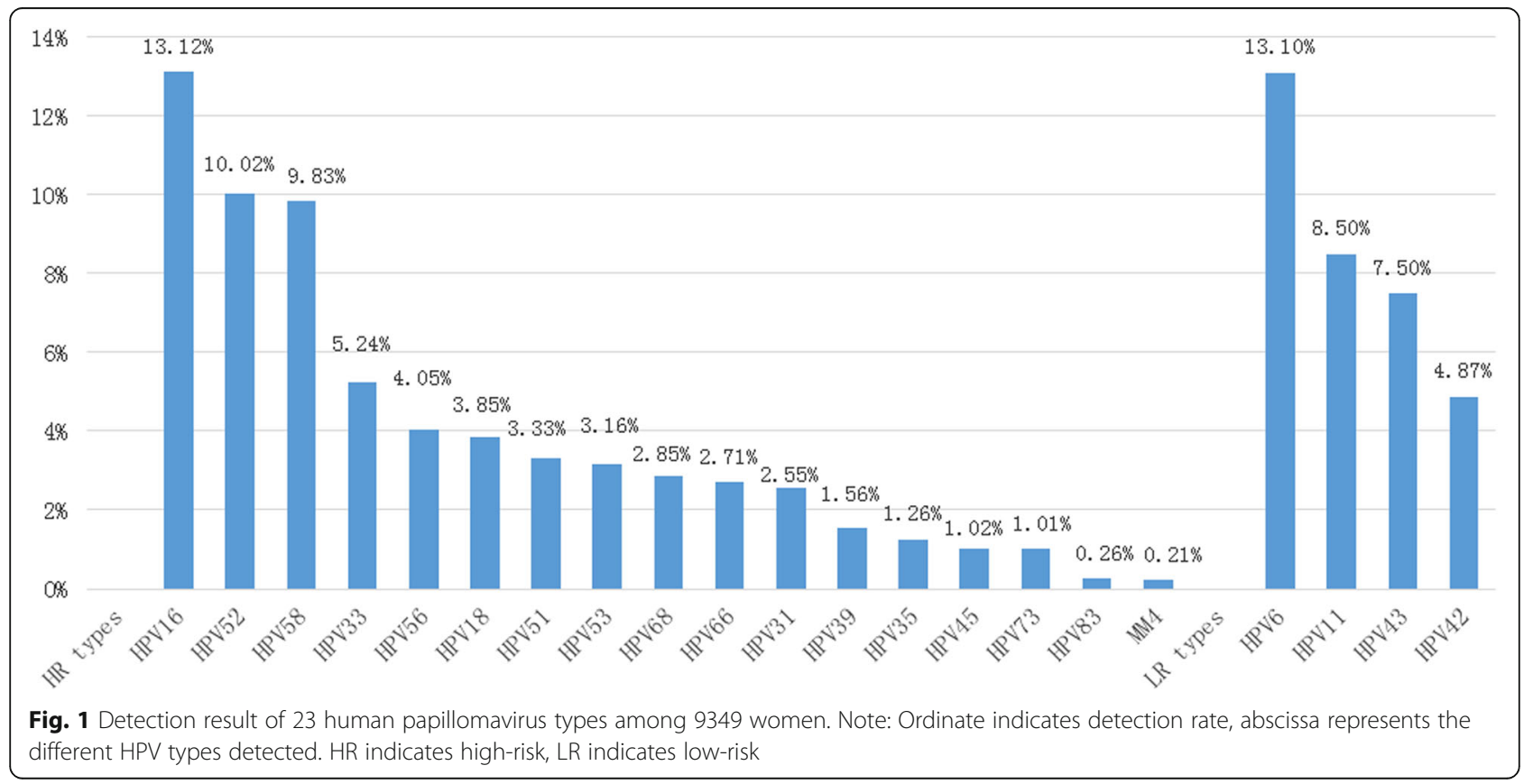


Table 1 Nucleotide sequence mutation at E6 of HPV6 isolates

\begin{tabular}{|c|c|c|c|c|c|c|c|c|c|}
\hline \multirow[t]{2}{*}{ Sequence pattern } & \multicolumn{7}{|c|}{ HPV6 E6 } & \multirow[t]{2}{*}{ Number of samples } & \multirow[t]{2}{*}{ Sub-lineages } \\
\hline & 40 & 120 & 150 & 222 & 264 & 291 & 372 & & \\
\hline Reference & G & $A$ & C & $A$ & $A$ & C & G & & \\
\hline HPV6E601 & . & $\mathrm{T}$ & . & . & . & . & A & 96 & $\mathrm{~A} 1$ \\
\hline HPV6E602 & $\mathrm{T}$ & $\mathrm{T}$ & . & . & . & . & A & 5 & A1 \\
\hline HPV6E603 & . & $\mathrm{T}$ & G & $\mathrm{T}$ & $\mathrm{T}$ & $\mathrm{T}$ & $A$ & 42 & B1 \\
\hline Reference AA & $D$ & $\mathrm{~T}$ & $\mathrm{H}$ & । & $\mathrm{T}$ & । & $A$ & & \\
\hline AA position & 14 & 40 & 50 & 74 & 88 & 97 & 124 & & \\
\hline AA mutations & Y & & Q & & . & . & . & & \\
\hline Secondary structure & $\mathrm{H}$ & & $\mathrm{H}$ & $\mathrm{H}$ & & S & & & \\
\hline
\end{tabular}

Note: The nucleotides conserved with respect to the reference sequence were marked with a dash (.), whereas a variation position was indicated by a letter. The " $\mathrm{S}$ " in the last row of the table means Strand, the " $\mathrm{H}$ " means Helix. The "AA" means amino acid. The " $D, T, H, I, A, Y, Q$ " in the seventh and ninth rows of the table represent aspartic acid, threonine, histidine, isoleucine, alanine, tyrosine, and glutanine, respectively. The numbers in the second row of the table mean nucleotide positions

sequence encoding $\beta$-strand (Table 2). No insertions, deletions, or premature stop codons were identified within the analyzed E7 variants. The (297 bp) E7 variants mainly belonged to sublineages A1 (65.73\%) and sublineages B1 (31.47\%) (Fig. 3). HPV6E701 was newly found.

\section{Structural analysis}

None of the identified variant sequences were predicted to introduce changes to the reference HPV6 E6 and E7 secondary structures (see Additional files 2, 3, 4 and 5). Overall, the secondary structure of the E6 protein was predicted to consist of $30.0 \%$ helix, $16.7 \%$ strand, and $53.3 \%$ coil elements, whereas that of the E7 protein was predicted to comprise $12.3 \%$ helix, $18.4 \%$ strand, and $69.3 \%$ coil elements. There was no obvious change in the predicted HPV6 E6 and E7 secondary structures.
The non-synonymous/synonymous rate ratio $(\mathrm{dN} / \mathrm{d} S)$, can measures selective pressure. When one non-synonymous mutation has advantage in fitness, it will rise to a higher rate than synonymous mutation, resulting in $\mathrm{dN} / \mathrm{dS}>1$. The variable $\mathrm{dN} / \mathrm{dS}$ ratios were tested among various lineages using the PAML 4.8 software. This program uses the Nei and Gojobori method of estimating synonymous substitutions, which is an unweighted pathway method. The first step in the procedure requires enumeration of the number of synonymous and nonsynonymous sites present at each codon, where each site may be both partially synonymous and non-synonymous. The next stage is to determine the number of synonymous and non-synonymous changes between each pair of aligned sequences, codon-by-codon. The results of the conducted selective pressure (i.e, variable $\mathrm{dN} / \mathrm{dS}$ ratio) analysis of the identified HPV6 E6 and E7 gene

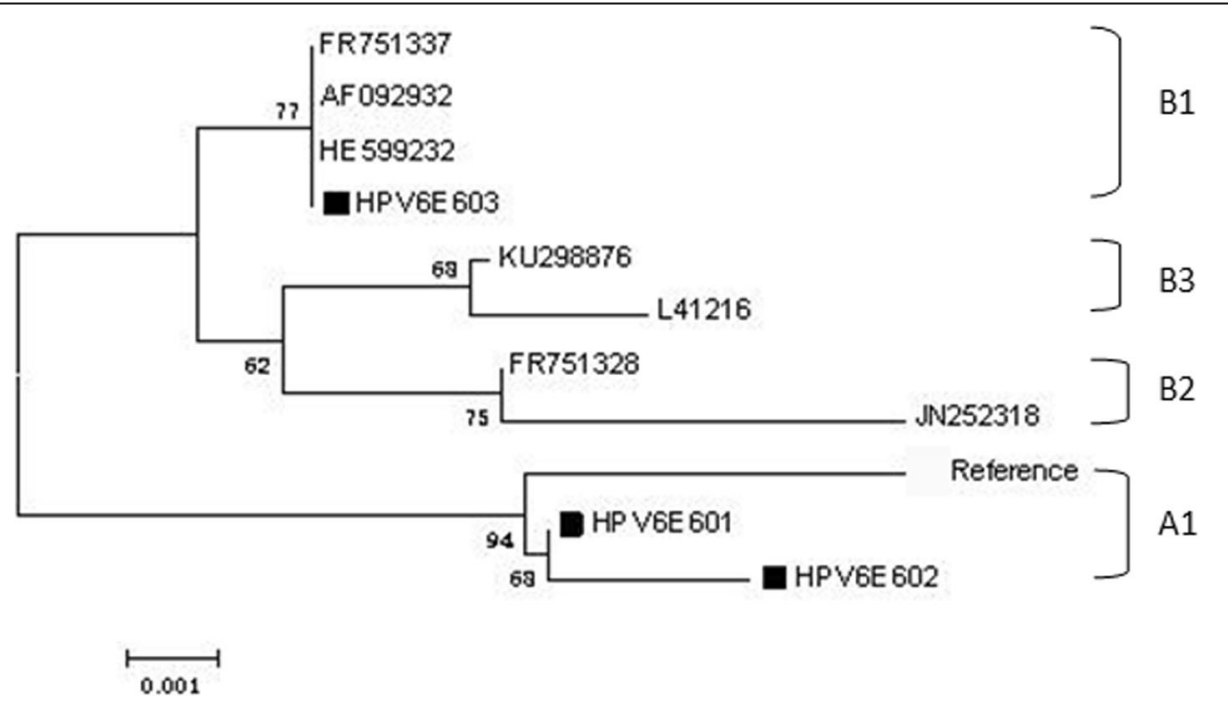

Fig. 2 The Maximum-likelihood tree of HPV6 E6 variants based on combined sequences. Note: Numbers above the branches indicate the bootstrap values that are greater than $60 \%$. The sample sequences are labeled in black square, others are standard sequences 
Table 2 Nucleotide sequence mutation at E7 of HPV6 isolates

\begin{tabular}{|c|c|c|c|c|c|c|c|}
\hline \multirow[t]{2}{*}{ Sequence pattern } & \multicolumn{5}{|c|}{ HPV6 E7 } & \multirow[t]{2}{*}{ Number of samples } & \multirow[t]{2}{*}{ Sub-lineages } \\
\hline & 10 & 11 & 100 & 155 & 294 & & \\
\hline Reference & $A$ & G & $G$ & $T$ & C & & \\
\hline HPV6E701 & G & A & A & A & A & 4 & \\
\hline HPV6E702 & . & . & . & A & A & 45 & B1 \\
\hline HPV6E703 & & . & & . & . & 94 & $\mathrm{~A} 1$ \\
\hline Reference AA & $\mathrm{R}$ & & $\mathrm{E}$ & $\mathrm{F}$ & T & & \\
\hline AA position & 4 & & 34 & 52 & 98 & & \\
\hline AA mutations & E & & K & Y & . & & \\
\hline Secondary structure & & & & S & & & \\
\hline
\end{tabular}

Note: the nucleotides conserved with respect to the reference sequence were marked with a dash (.), whereas a variation position was indicated by a letter. The " $\mathrm{S}$ " in the last row of the table means Strand, the " $\mathrm{H}$ " means Helix. The " $A A^{\prime}$ " means amino acid. The "R, E, F, T, K, $Y$ " in the seventh and ninth rows of the table represent arginine, glutamic acid, phenylalanine, threonine, lysine and tyrosine, respectively. The numbers in the second row of the table mean nucleotide positions

sequences are summarized in Tables 3 and 4 . No positively selected sites were identified within the $E 6$ sequence; however, in contrast, the E7 variant sites $\mathrm{R} 4 \mathrm{E}$ (arginine to glutamic acid), E34K (glutamic acid to lysine), and F52Y (phenylalanine to tyrosine), were predicted to be subjected to positive selection.

The analysis of sequence results and reference sequence Comparation between the results of our sequence with the reference sequence has been listed in the Table 5. For E6, the mutations A120T, C150G, A264T, and C291T were discovered in all sequence listed. The mutation G40 $\mathrm{T}$ was only found in HPV6vc (Table 5). Point mutations altered the E6 AA sequence of non-prototypic isolates, which contained specific mutations at nucleotide positions 150 (H50Q). AA exchanges identified at nucleotide positions 40 (D14Y) were additional characteristic features.
The mutation at nucleotide positions 55 (T19S) was not found in this study. For E7, A10G, G11A, and G100A were proved as new mutations in this study. The mutations C156T and A262G were not discovered in the research. All sequence listed contain the mutation C294A. Point mutations altered the E7 AA sequence of nonprototypic isolates, which contained specific mutations at nucleotide positions 155 (F52K). AA exchanges identified at nucleotide positions 10, 11 (R4E) and 100(E34K) were additional characteristic features. The mutation at nucleotide positions $262(\mathrm{D} 88 \mathrm{~N})$ was not found in this study.

\section{Discussion}

E6 and E7 are essential HPV E-gene products, they target $\mathrm{P} 53$ and retinoblastoma (Rb) tumuor-suppressor proteins, respectively. The degradation of $\mathrm{PRb}$ can initiate abnormal cell replication; the inhibition of $p 53$ can

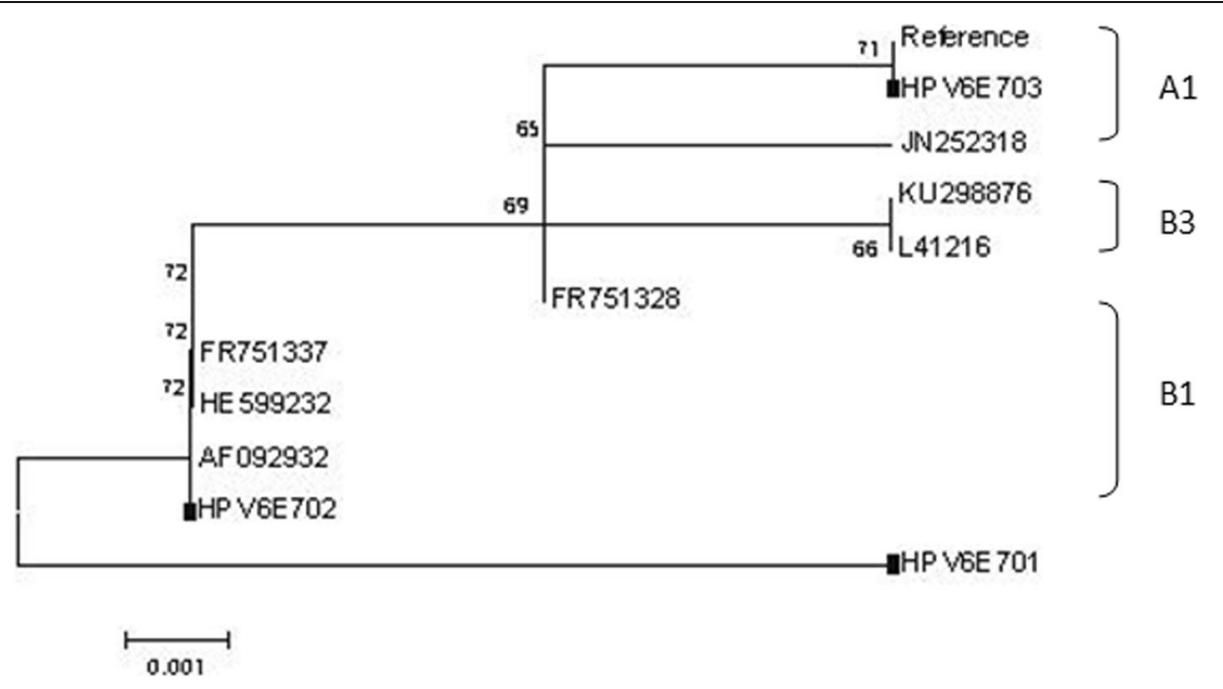

Fig. 3 The Maximum-likelihood tree of HPV6 E7 variants based on combined sequences. Note: Numbers above the branches indicate the bootstrap values that are greater than $60 \%$. The sample sequences are labeled in black square, and others are standard sequences 
Table 3 Site-specific tests for positive selection on HPV6 E6

\begin{tabular}{lllll}
\hline Models & $\mathrm{InL}$ & Estimates of parameters & $2 \Delta l$ & Positively selected sites \\
\hline M7 & -662.94 & $p=12.39037 \mathrm{q}=99.00000$ & NA \\
M8 & -662.94 & $\begin{array}{l}\mathrm{p} 0=0.99999 p=12.38952 \mathrm{q}=99.00000 \\
(\mathrm{p} 1=0.00001) \mathrm{w}=1.00000\end{array}$ & $0 \mathrm{P}=1$ & NONE \\
\hline
\end{tabular}

Note: InL, the log-likelihood difference between the two models. $2 \Delta \mathrm{l}$, twice the log-likelihood difference between the two models $(P<0.05$ was considered statistically significant). NA means not applicable. M7 means specifies the model of nucleotide substitution (REV); M8 means specifies the model of nucleotide substitution (UNREST)

cause abnormal replication cell to lose control. The HPV genome can only be replicated along with the replication of the host genome. Therefore, E6 and E7 are especially important in HPV lifecycle. The present study sequenced the E6 and E7 ORFs in 143 HPV6 isolates from patients in Southwest China, to assess the HPV6 genetic diversity and evolution characteristics within this population, and help further identify specific HPV6 variants.

HPV6 was the second prevalent HPV type and the most prevalent low risk HPV type in southwest China, indicating that HPV6 is highly adaptable to the environment in southwest China compare with most HPV types that are not so prevalent.

Seven E6 sequence variants were identified, two of which induced corresponding E6 amino acid changes. One of these, H50Q (His to Gln), was identified in $30.08 \%$ of the analyzed HPV6 sequences, the amino acid at position 50 of prototype HPV6 E6 may be located centrally between the two internal Zn-binding motifs, which is important for the E6 protein stability [22]. These variants have not been discovered previously in Southwest China, and their functional impact requires further analysis. Overall, the HPV6 E6 sequence was shown to be highly conserved within the analyzed patient samples, which exhibited only two main genotypes, HPV6E601 and HPV6E603, that represented 67.13\% (96/ $143)$ and $29.37 \%(42 / 143)$ of the samples, respectively. Likewise, five $E 7$ variants were identified, four of which exhibited a corresponding E7 amino-acid change. The most common E7 nucleotide mutations were T155A and C294A. Notably, T155A mutation affected 34.27\% (49/143) patients, and caused a F52Y (Phe to Tyr) amino acid conversion that was predicted impact the protein's secondary structure. Furthermore, the E7 sequence also was proved to be highly conserved within the patient specimens, comprising only two main genotypes HPV6E702 and HPV6E703 that were exhibited by $31.47 \%(45 / 143)$ and $65.73 \%(94 / 143)$ of the analyzed patients, respectively, and the sequence of HPV6E703 is in conformity with the reference (X00203). The fact that both sequences were so highly conserved supports the hypothesis that they play vital roles in HPV6 structure and function [23, 24], consistent with previous studies by Dartmann [25]. Thus, they are likely also promising targets for HPV6 primer design and diagnostic detection. Compared to deadly cancerogenic high risk HPV, low risk HPV is able to get along better with the host; low risk HPV is more likely to be ignored in clinical treatment and prevention. HPV6 E6 and E7 were much more conserved than main high risk HPV types (like 16, 33, 53 and 58), may indicating low risk HPV has lower evolutionary pressure in gene level [20, 21, 26].

Structurally, some of the detected mutations affected amino acids at critical positions related to known biological functions. In E6, one (I97) and three (D14Y, $\mathrm{H} 50 \mathrm{Q}$, and I74) mutations were found to affect the $\beta$ strand and $\alpha$-helix-encoding regions, respectively, that are critical for structural stability. In E7, only one mutation (F52Y) was detected may affect the $\beta$-strand-encoding region. The nucleotides as positions 58,61, 91, and 94 in the E7 protein sequence have been previously shown to act as zinc binding sites [27], but no mutations were identified by the present study to occur in these positions, nor in the consensus LXCXE RB1-binding site (positions 22-26), or zinc-binding motif.

Notably, the present study is first to conduct an analysis to assess whether the E6 and E7 sequences are subject to positive selection in Southwest China. The main characteristic of positive selection is that it causes an

Table 4 Site-specific tests for positive selection on HPV6 E7

\begin{tabular}{lllll}
\hline Models & InL & Estimates of parameters & $2 \Delta l$ & Positively selected sites \\
\hline M7 & -436.09 & $p=1.36732 q=0.00500$ & NA \\
M8 & -433.89 & $\begin{array}{l}p 0=0.93931 p=0.00500 q=8.82347 \\
(p 1=0.06069) \mathrm{W}=29.56283\end{array}$ & $4.40 P<0.01$ & $4 \mathrm{R}, 34 \mathrm{E}, 52 \mathrm{~F}$ \\
\end{tabular}

Note: InL, the log-likelihood difference between the two models. $2 \Delta \mathrm{l}$, twice the log-likelihood difference between the two models $(\mathrm{P}<0.05$ was considered statistically significant); NA means not applicable. M7 means specifies the model of nucleotide substitution (REV); M8 means specifies the model of nucleotide substitution (UNREST) 
Table 5 Sequence results compared to reference sequences

\begin{tabular}{|c|c|c|c|c|c|c|c|c|c|c|c|c|c|c|c|c|c|c|}
\hline \multirow[t]{2}{*}{ Sequence } & \multicolumn{11}{|c|}{ HPV6 E6 } & \multicolumn{7}{|c|}{ HPV6 E7 } \\
\hline & 40 & 55 & 120 & 150 & 159 & 222 & 264 & 279 & 291 & 372 & 378 & 10 & 11 & 100 & 155 & 156 & 262 & 294 \\
\hline Reference & $G$ & A & A & $C$ & C & $A$ & A & $T$ & C & $G$ & C & $A$ & $G$ & $G$ & $T$ & $C$ & $A$ & $C$ \\
\hline HPV6VC & $\mathrm{T}$ & & T & G & & $\mathrm{T}$ & $\mathrm{T}$ & . & $\mathrm{T}$ & A & & G & A & A & A & & . & $A$ \\
\hline KU298876 & & . & T & G & . & C & $\mathrm{T}$ & & $T$ & A & $\mathrm{T}$ & & . & . & & . & G & A \\
\hline HE599232 & . & & T & G & . & T & $\mathrm{T}$ & . & $\mathrm{T}$ & A & . & . & & . & $A$ & . & . & A \\
\hline FR751328 & & T & $\mathrm{T}$ & G & . & $C$ & $\mathrm{~T}$ & & $\mathrm{~T}$ & A & & & . & . & & . & . & A \\
\hline FR751337 & & . & T & G & . & $T$ & $\mathrm{~T}$ & & $T$ & A & & & . & . & $A$ & . & & A \\
\hline AF092932 & & . & $\mathrm{T}$ & G & . & T & $\mathrm{T}$ & & $\mathrm{T}$ & A & & . & . & . & A & 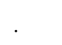 & . & A \\
\hline L41216 & & . & T & G & . & C & $\mathrm{T}$ & & $T$ & & $\mathrm{~T}$ & & . & . & & . & G & A \\
\hline JN252318 & & $T$ & T & G & T & C & T & G & $T$ & A & . & & . & . & & $T$ & . & A \\
\hline Reference AA & $\mathrm{D}$ & $\mathrm{T}$ & & $\mathrm{H}$ & & & & & & & & $\mathrm{R}$ & & E & $\mathrm{F}$ & & $D$ & \\
\hline AA position & 14 & 19 & & 50 & & & & & & & & 4 & & 34 & 52 & & 88 & \\
\hline AA mutations & Y & S & & Q & & & & & & & & E & & K & K & & N & \\
\hline
\end{tabular}

Note: The nucleotides conserved with respect to the reference sequence were marked with a dash (.), whereas a variation position was indicated by a letter. The numbers in the second row of the table mean nucleotide positions. "HPV6vc" includes all mutations of the sequenced samples. AA means amino acid

unusually rapid rise in allele frequency, thereby enabling a species to adapt rapidly to environmental changes [28]. The selective pressure analysis showed that all the sites that evolved under positive selection were common nonsynonymous mutations, indicating that the positively selected variations beneficial for HPV6 to accommodate their environments are wide-spread. Moreover, remarkably, the positive sites R4E, E34K, and F52Y were observed in HPV6 E7, they may have evolutionary significance in making HPV6 adaptive to their environments.

Previous studies revealed the existence of two variant lineages (lineage $\mathrm{A}$, lineage $\mathrm{B}$ ) and five variant sub-lineages (sub-lineage B1, B2, B3, B4, B5) [29] among HPV6 variants. The conducted phylogenetic analyses showed that $97.56 \%$ of the $E 6$ and $E 7$ sequences within the patient specimens belonged to sub-lineage A1 (Reference, HPV6b) and sub-lineage B1 (HE599232, LP243) [29]. Lineage A has been shown to predominate in Asia; in contrast, lineage $\mathrm{B}$ is distributed globally [30, 31], and consists mainly of variants from sub-lineage B1. Given its global distribution, lineage B1 may represent the oldest HPV6 sub-lineage, and likely disseminated during early human evolution. This may have enabled it to migrate to different regions of the world prior to the emergence of other HPV6 sub-lineages [29], and for E7, a new sub-lineage was found, however, only three samples were detected, and therefore, it requires further study.

Certain strengths of this study were that all clinical samples were collected from the Southwest region of China which had a strong regional representation and several new mutations were discovered, which will provide real and valid data for development of therapeutic vaccines for affected people in the Southwest China.
Thus, the results of the present study significantly expand the current knowledge of HPV6 genetic diversity in Southwest China, and also provide a valuable resource for future studies of HPV6 epidemiology, evolution, function, pathogenesis, and use as a therapeutic target. However, the cellular level research is required, and a series of cellular experiments about the mutations should be designed for future studies.

\section{Conclusions}

HPV6 was very prevalent in southwest China, both the HPV6 E6 and E7 sequences were highly conserved within the analysed patient samples in southwest China, may indicating that the low risk HPV6 can adapt to the environment well without much evolution.

\section{Supplementary information}

Supplementary information accompanies this paper at https://doi.org/10. 1186/s12985-019-1221-x.

Additional file 1: Table S1. HPV6 E6 and E7 primers. (DOC $32 \mathrm{~kb}$ )

Additional file 2: HPV6 E6 reference sequence predicted secondary structure. Secondary structure within the reference sequence of HPV6 E6 protein. (PDF $14 \mathrm{~kb})$

Additional file 3: HPV6 E6 variation sequence predicted secondary structure. Secondary structure within the variation sequence of HPV6 E6 protein. Variations of HPV6E601, HPV6E602 and HPV6E603 were integrated into one sequence to predict. (PDF $15 \mathrm{~kb}$ )

Additional file 4: HPV6 E7 reference sequence predicted secondary structure. Secondary structure within the reference sequence of HPV6 E7 protein. (PDF $11 \mathrm{~kb}$ )

Additional file 5: HPV6 E7 variation sequence predicted secondary structure. Secondary structure within the variation sequence of HPV6 E7 protein. Variations of HPV6E701, HPV6E702 and HPV6E703 were integrated into one sequence to predict. (PDF $11 \mathrm{~kb}$ ) 


\section{Abbreviations}

AA: Amino acid; BEB: Bayes empirical Bayes; HPV: Human papillomavirus; MEGA: Molecular evolutionary genetics analysis version; ORF: Open reading frame; PAML: Phylogenetic analyses by maximun likelihood; $\mathrm{Rb}$ : Retinoblastoma

\section{Acknowledgments}

We thank for Affiliated Hospital of Zunyi Medical University, the Angel Women's and Children's Hospital, Sichuan Reproductive Health Research Center Affiliated Hospital, and Chengdu Western Hospital Maternity Unit Hospital, and some additional hospitals.

\section{Authors' contributions}

ZC, QL and XM conceived and designed the study. ZC, QL, JH, ZL, FY, XM and ZC performed the experiments. ZC and QL analyzed the data. ZC and QL wrote the paper. All authors read and approved the final manuscript.

\section{Funding}

This reseach was funded by Affiliated Hospital of Zunyi Medical University (number 201808).

\section{Availability of data and materials}

All data generated or analyzed during this study are included in this published article and GeneBank.

\section{Ethics approval and consent to participate}

The study was approved by the Ethics Committee of Affiliated Hospital of Zunyi Medical University, the approval number was ZYFYLS2018(81). Before sample collection, a written informed consent was obtained from all the patients or their guardians, and patient/study subject privacy was carefully protected.

\section{Consent for publication}

Not applicable.

\section{Competing interests}

The authors declare that they have no competing interests.

\section{Author details}

${ }^{1}$ Department of Laboratory medicine, Affiliated Hospital of Zunyi Medical University, Zunyi, Guizhou, People's Republic of China. ${ }^{2}$ Bio-resource Research and Utilization Joint Key Laboratory of Sichuan and Chongqing, Chongqing, Sichuan, People's Republic of China. ${ }^{3}$ Department of Information Technology, Affiliated Hospital of Zunyi Medical University, Zunyi, Guizhou, People's Republic of China. ${ }^{4}$ Department of Thoracic Surgery, Affiliated Hospital of Zunyi Medical University, Zunyi, Guizhou, People's Republic of China.

Received: 1 July 2019 Accepted: 5 September 2019

\section{Published online: 12 September 2019}

\section{References}

1. Doorbar J, Griffin H. Intrabody strategies for the treatment of human papillomavirus-associated disease [J]. Expert Opin Biol Ther. 2007;7(5):67789.

2. Cheah PL, Looi LM. Biology and pathological associations of the human papillomaviruses: a review [J]. Malays J Pathol. 1998;20(1):1-10.

3. Singh M, Thakral D, Kar HK, et al. Distinct clinico-immunological profile of patients infected with human papilloma virus genotypes 6 and 11[J]. Virus Dis. 2017;28(2):200-4

4. De Koning MNC, Quint KD, Bruggink SC, et al. High prevalence of cutaneous warts in elementary school children and the ubiquitous presence of wart-associated human papillomavirus on clinically normal skin [J]. $\mathrm{Br}$ J Dermatol. 2015;172(1):196-201.

5. Stone A, Potton A. Emotional responses to disfigured faces and disgust sensitivity: an eye-tracking study [J]. J Health Psychol. 2017; 1359105317692856.

6. DiLorenzo TP, Tamsen A, Abramson AL, et al. Human papillomavirus type $6 a$ DNA in the lung carcinoma of a patient with recurrent laryngeal papillomatosis is characterized by a partial duplication [J]. J Gen Virol. 1992; 73(2):423-8.
7. Bercovich JA, Centeno CR, Aguilar OG, et al. Presence and integration of human papillomavirus type 6 in a tonsillar carcinoma [J]. J Gen Virol. 1991;72(10):2569-72

8. Zarod AP, Rutherford JD, Corbitt G. Malignant progression of laryngeal papilloma associated with human papilloma virus type 6 (HPV-6) DNA [J]. J Clin Pathol. 1988;41(3):280-3.

9. Venuti A, Manni V, Morello R, et al. Physical state and expression of human papillomavirus in laryngeal carcinoma and surrounding normal mucosa [J]. J Med Virol. 2000;60(4):396-402.

10. Garcã-A-Vallvã S, Alonso A, Bravo IG. Papillomaviruses: different genes have different histories [J]. Trends Microbiol. 2005;13(11):514-21.

11. Mcmurray HR, Nguyen D, Westbrook TF, et al. Biology of human papillomaviruses.[J]. Int J Exp Pathol. 2010;82(1):15-33.

12. Raiol WT, Amorim PSD, et al. Genetic variability and phylogeny of the highrisk HPV-31, $-33,-35,-52$, and -58 in Central Brazil [J]. J Med Virol. 2010; 81(4):685-92.

13. Thomas JT, Hubert WG, Ruesch MN, et al. Human papillomavirus type 31 oncoproteins E6 and E7 are required for the maintenance of episomes during the viral life cycle in normal human keratinocytes [J]. Proc Natl Acad Sci. 1999;96(15):8449-54.

14. Buchan DWA, Minneci F, Nugent TCO, et al. Scalable web services for the PSIPRED protein analysis workbench [J]. Nucleic Acids Res. 2013;41(W1):W349-57.

15. Tamura K, Stecher G, Peterson D, et al. MEGA6: molecular evolutionary genetics analysis version 6.0[J]. Mol Biol Evol. 2013;30(12):2725-9.

16. Nei M, Gojobori T. Simple methods for estimating the numbers of synonymous and nonsynonymous nucleotide substitutions [J]. Mol Biol Evol. 1986:3(5):418-26.

17. Yang Z. PAML 4: phylogenetic analysis by maximum likelihood [J]. Mol Biol Evol. 2007:24(8):1586-91.

18. Hamza AA, Robene-Soustrade I, Jouen E, et al. MultiLocus sequence analysis- and amplified fragment length polymorphism-based characterization of xanthomonads associated with bacterial spot of tomato and pepper and their relatedness to Xanthomonas species [J]. Syst Appl Microbiol. 2012:35(3):183-90.

19. Hwang T. Detection and typing of human papillomavirus DNA by PCR using consensus primers in various cervical lesions of Korean women [J]. J Korean Med Sci. 1999;14(6)

20. Chen $Z$, Jing $Y$, Wen $Q$, et al. E6 and E7 gene polymorphisms in human papillomavirus Types-58 and 33 identified in Southwest China [J]. PLoS One. 2017;12(1):e0171140.

21. Chen Z, Jing Y, Wen Q, et al. L1andL2gene polymorphisms in HPV-58 and HPV-33: implications for vaccine design and diagnosis [J]. Virol J. 2016;13(1):167.

22. Barbosa MS, Lowy DR, Schiller JT. Papillomavirus polypeptides E6 and E7 are zinc-binding proteins [J]. J Virol. 1989;63(3):1404-7.

23. Donne AJ, Hampson L, Homer JJ, et al. The role of HPV type in recurrent respiratory papillomatosis [J]. Int J Pediatr Otorhinolaryngol. 2010;74(1):0-14.

24. Hummel M, Hudson JB, Laimins LA. Differentiation-induced and constitutive transcription of human papillomavirus type $31 \mathrm{~b}$ in cell lines containing viral episomes [J]. J Virol. 1992:66(10):6070-80.

25. Dartmann K, Schwarz E, Gissmann L, et al. The nucleotide sequence and genome organization of human papilloma virus type 11[J]. Virology. 1986;151(1):124-30.

26. Cao M, Chenzhang Y, Ding $X$, et al. Genetic variability and lineage phylogeny of human papillomavirus Type-16 and -53 based on the E6, E7, and L1 genes in Southwest China.[J]. Gene. 2016;592(1):49-59.

27. Chemes LB, Camporeale G, Sánchez IE, et al. Cysteine-rich positions outside the structural zinc motif of human papillomavirus E7 provide conformational modulation and suggest functional redox roles [J]. Biochemistry. 2014;53(10):1680-96.

28. lyengar VK, Reeve HK, Eisner T. Paternal inheritance of a female moth \"s mating preference [J]. Nature, 2002, 419(6909):830-832

29. Jelen M M, Chen Z, Kocjan B J, et al. Global genomic diversity of human papillomavirus 6 based on 724 isolates and 190 complete genome sequences[J]. Journal of virology. 2014;88(13):7307-7316.

30. Danielewski JA, Garland SM, Jenny MC, et al. Human papillomavirus type 6 and 11 genetic variants found in 71 Oral and Anogenital epithelial samples from Australia [J]. PLoS One. 2013;8(5):e63892.

31. Kocjan BJ, Poljak M, Cimerman M, et al. Prevaccination genomic diversity of human papillomavirus genotype 6 (HPV 6)[J]. Virology. 2009;391(2):274-83.

\section{Publisher's Note}

Springer Nature remains neutral with regard to jurisdictional claims in published maps and institutional affiliations. 